\title{
Enhancing WS-BPEL Dynamic Invariant Generation Using XML Schema and XPath Information
}

\author{
Manuel Palomo-Duarte, Antonio García-Domínguez, \\ and Inmaculada Medina-Bulo \\ Universidad de Cádiz, Escuela Superior de Ingeniería, c/Chile 1, CP 11003 Cádiz, \\ Spain \\ \{manuel.palomo, antonio.garciadominguez, inmaculada.medina\}@uca.es
}

\begin{abstract}
The dynamic and asynchronous nature of OASIS WS-BPEL 2.0 standard language for web service composition presents a challenge for traditional white-box testing techniques. Takuan is a tool that can help with this problem. It analyzes execution logs of a WS-BPEL process in a real-world WS-BPEL engine to dynamically generate composition invariants. Nevertheless, it suffered some performance issues when handling a large number of variables with complex multidimensional content. We present two techniques implemented to automatically alleviate these issues: dynamic analysis of XPath expressions to avoid inferring nonsensical invariants, and discarding invariants enforced by the XML Schema. We present practical results supporting our work and comment the impact and applicability of these techniques beyond WS-BPEL itself.
\end{abstract}

Keywords: Web services, service composition, WS-BPEL, white-box testing, dynamic invariant generation.

\section{Introduction}

The OASIS WS-BPEL (Web Service Business Process Execution Language) 2.0 standard allows programing in the large using web service compositions. However, it presents a challenge [1] for traditional white-box testing techniques, due to the inclusion of WS-specific instructions not found in most programming languages (like those for fault and compensation handling).

Automatic invariant generation [2] has proved to be a successful technique to assist in white-box testing of programs written in imperative languages. Let us note that, throughout this work, invariant and likely invariant are understood (as in most related works) in their broadest sense: properties which hold always or in the specified test cases at a certain program point, respectively.

We have implemented Takuan [3, an open-source system which dynamically generates likely invariants from actual WS-BPEL composition execution logs. Its automatic workflow takes a WS-BPEL process definition and a test suite specification and outputs a collection of invariants which hold at certain program points in every test case. 
This paper describes two techniques which aim to reduce Takuan's resource usage (increasing its scalability to larger compositions) as well as the number of uninteresting invariants in its output. We first study their impact and applicability on WS-BPEL and then point out other languages with similar issues.

\section{Improving Invariant Generation with XPath}

Takuan results were promising [3, but even for slightly complex WS-BPEL compositions there was very high memory and CPU usage depending on the options used. For example, analyzing 7 test cases in a meta-search composition could take over 7 minutes and use over $800 \mathrm{MiB}$ of RAM [4].

The reason was the large number of invariants comprising pairs and triples of variables that had to be checked, many of them nonsensical. For example, the age of an applicant to a loan and its amount can be both integers, but there is no need to waste computational resources trying to relate them in an invariant.

We avoided it by labeling each variable with a so-called comparability index: an integer which marks a set of variables as belonging to a specific semantic type, such as a monetary sum or a vector size. This way Daikon (the dynamic invariant generator internally used by Takuan) only infers invariants relating variables that have the same index, avoiding the combinatorial explosion and will even discard uninteresting program points and, optionally, unused variable fields.

We had to extend all steps of the Takuan framework to implement this functionality. Discarding unused variable fields is suitable for any language in which variables from outer scopes are directly used only in a few key program points and control flow is highly structured.

\section{$3 \quad$ Filtering Redundant Invariants}

In our experience using Takuan [4, we have observed that a considerable number of the invariants only repeat constraints which are already enforced by the XML Schema declarations of the types used in the composition.

For instance, if XML Schema tells us that a variable has to be in the range $[0,1]$ an invariant confirming it would only add noise in the resulting invariant list. In fact, if that information relates to a WS-BPEL multidimensional variable that is later mapped into $n$ unidimensional Takuan variables by matrix flattening, we will have avoided generating not one, but $n$ invariants.

We implemented this technique extending Daikon itself, but keeping it as language-independent as possible. Other languages have type systems that include similar information to some parts of XML Schema: statically sized arrays and matrices in $\mathrm{C} / \mathrm{C}++$, fixed length strings in FORTRAN, or VARCHAR ( $\mathrm{N}$ ) fields in SQL, for instance.

\section{Results}

We have studied the impact that each of these two techniques have in the analysis of the meta-search engine composition discussed in [4] with the same test suite. 
Table 1. Input, output and performance metrics for each combination of techniques

\begin{tabular}{|c|c|c|c|c|c|c|}
\hline Mapping $^{a}$ & Techniques $^{b}$ & P. points ${ }^{c}$ & Variables & Memory $^{d}$ & Time $^{e}$ & Invariants \\
\hline \multirow{6}{*}{ Slicing } & None & \multirow[b]{2}{*}{64} & \multirow[b]{2}{*}{17,404} & 656.74 & 409.98 & 30,399 \\
\hline & $\mathrm{X}$ & & & 646.57 & 400.15 & 21,793 \\
\hline & $\mathrm{C}$ & \multirow{4}{*}{48} & \multirow{2}{*}{14,148} & 561.63 & 416.72 & 27,089 \\
\hline & $\mathrm{CX}$ & & & 579.81 & 401.69 & 18,358 \\
\hline & $\mathrm{CF}$ & & \multirow{2}{*}{1,398} & 25.25 & 72.96 & 2,135 \\
\hline & $\mathrm{CXF}$ & & & 24.54 & 75.03 & 1,559 \\
\hline \multirow{6}{*}{ Flattening } & None & \multirow{2}{*}{64} & \multirow{2}{*}{11,412} & 291.11 & 162.00 & 18,658 \\
\hline & $\mathrm{X}$ & & & 280.96 & 173.40 & 18,654 \\
\hline & $\mathrm{C}$ & \multirow{4}{*}{48} & \multirow[b]{2}{*}{9,036} & 261.01 & 179.39 & 16,718 \\
\hline & $\mathrm{CX}$ & & & 264.54 & 163.90 & 16,714 \\
\hline & $\mathrm{CF}$ & & \multirow{2}{*}{710} & 11.18 & 52.29 & 942 \\
\hline & $\mathrm{CXF}$ & & & 12.29 & 55.61 & 940 \\
\hline
\end{tabular}

${ }^{a}$ See [4] for descriptions of these mappings.

${ }^{b} \mathrm{C}$ : comparability indices, X: XML Schema invariant suppression, F: unused variable filtering.

${ }^{c}$ Program points with no XPath expressions therein are removed during comparability analysis.

${ }^{d}$ Maximum usage of the JVM heap by Daikon, measured in MiB.

${ }^{e}$ Time required by Takuan's analysis step, including preprocessing, measured in seconds.

We have used each of them separately and in combination. Table 1 shows the computational resources (time and space) required for their analysis, and the length of the invariant list produced by each combination.

The maximum memory used at some point by the preprocessor Perl scripts remains approximately the same at $193 \mathrm{MiB}$ for every entry in the table. The test environment consisted of a machine fitted with a dual-core Intel Core Duo T2250 CPU, with $1 \mathrm{GiB}$ of DDR2 533MHz RAM and a 80GB 5400rpm HDD. The base system used was a standard GNU/Linux Ubuntu 8.04.1 distribution installation, with its 2.6.24-19-generic default kernel. The active processes during the test suite were mainly those created by the components of Takuan: the Sun 6.0 JRE (with a maximum JVM heap size of $800 \mathrm{MiB}$ ), Apache Ant 1.7.0, Perl 5.8.8, Daikon 4.3.4, ActiveBPEL 4.1 and BPELUnit 1.0. During its execution there were no other processes consuming significant CPU time, memory space, or disk throughput.

In our quantitative and qualitative analysis, we see that performing the comparability analysis while only filtering program points does not affect performance as much as expected, but it does improve the quality of the invariants inferred. Likewise, using the information encoded in the XML Schema only 
presents a minor performance improvement if any, but the number of invariants produced is reduced in some cases over $35 \%$.

In contrast, performance is drastically improved when unused variable fields at each program point are filtered, reducing on average the number of variable fields to be checked over $90 \%$ and the running time to a third.

\section{Conclusions and Future Work}

Takuan is an automated framework for dynamic invariant generation in WSBPEL compositions. In this paper we have shown two techniques which have been recently implemented into Takuan to reduce computational costs and improve the usefulness and conciseness of the list of invariants inferred. They can be applied to other languages, as little about them is exclusive to WS-BPEL.

Our future work will be validating our findings so far with more complex WSBPEL process compositions. Once Takuan obtains a satisfying balance between performance and invariant quality, we will focus on the relation between the quality of the invariants generated and the test suite used.

\section{Acknowledgments}

This paper has been funded by the Department of Education and Science (Spain) and FEDER funds under the National Program for Research, Development and Innovation. Project SOAQSim (TIN2007-67843-C06-04).

\section{References}

1. Bucchiarone, A., Melgratti, H., Severoni, F.: Testing service composition. In: Proceedings of the 8th Argentine Symposium on Software Engineering (ASSE 2007) (2007)

2. Ernst, M.D., Cockrell, J., Griswold, W.G., Notkin, D.: Dynamically discovering likely program invariants to support program evolution. IEEE Transactions on Software Engineering 27(2), 99-123 (2001)

3. Palomo-Duarte, M., García-Domínguez, A., Medina-Bulo, I.: Takuan: A dynamic invariant generation system for WS-BPEL compositions. In: ECOWS 2008: Proceedings of the 2008 Sixth European Conference on Web Services, Washington, DC, USA, pp. 63-72. IEEE Computer Society, Los Alamitos (2008)

4. Palomo-Duarte, M., García-Domínguez, A., Medina-Bulo, I.: Improving Takuan to analyze a meta-search engine WS-BPEL composition. In: SOSE 2008: Proceedings of the 2008 IEEE International Symposium on Service-Oriented System Engineering, Washington, DC, USA, pp. 109-114. IEEE Computer Society, Los Alamitos (2008) 